\title{
Lightning Current Measurement with Fiber-Optic Sensor
}

\author{
Invited Paper
}

\author{
Truong X. Nguyen, \\ Jay J. Ely and George N. Szatkowski \\ NASA Langley Research Center \\ Hampton, VA 23681 U.S.A. \\ truong.x.nguyen@nasa.gov
}

\author{
Carlos T. Mata and Angel G. Mata \\ ESC - Kennedy Space Center, FL 32899 U.S.A. \\ Gary P. Snyder \\ NASA - Kennedy Space Center, FL 32899 U.S.A.
}

\begin{abstract}
A fiber-optic current sensor is successfully developed with many potential applications for electric current measurement. Originally developed for in-flight lightning measurement, the sensor utilizes Faraday Effect in an optical fiber. The Faraday Effect causes linear light polarization in a fiber to rotate when the fiber is exposed to a magnetic field. The polarization change is detected using a reflective polarimetric scheme. Forming fiber loops and applying Ampere's law, measuring the total light rotation results in the determination of the total current enclosed. The sensor is conformable to complex structure geometry. It is also non-conductive and immune to electromagnetic interference, saturation or hysteresis. Installation is non-intrusive, and the sensor can be safely routed through flammable areas. Two similar sensor systems are described in this paper. The first system operates at $1310 \mathrm{~nm}$ laser wavelength and is capable of measuring approximately $300 \mathrm{~A}-300 \mathrm{kA}$, a 60 $\mathrm{dB}$ range. Laboratory validation results of aircraft lighting direct and in-direct effect current amplitudes are reported for this sensor. The second system operates at $1550 \mathrm{~nm}$ wavelength and can measure about 400 A - $400 \mathrm{kA}$. Triggered-lightning measurement data are presented for this system. Good results are achieved in all cases.
\end{abstract}

Keywords-lightning; Faraday Effect; fiber-optic; current; lightning

\section{INTRODUCTION}

Accurate characterization of in-flight lightning return stroke current is important in developing aircraft test requirements and protection against lightning direct and indirect effects. Accurate measurements may also help to infer aircraft damages immediately after a strike from which appropriate actions are determined. Sensors used in previous flight efforts to characterize lightning parameters [1,2] either required significant and expensive aircraft structure modifications (i.e. resistive shunt, I-dot sensor), or were unable to directly measure total current (i.e. magnetic field sensors). Timederivative sensors cannot measure low frequency components near DC. Rogowski coil and current transformer are not practical for external installations due to strict requirements on safety, weight, and aircraft aerodynamic performance.

This paper describes a fiber-optic current sensor based on Faraday (Rotation) Effect that can directly measure current on aircraft structures and is highly suitable for most aircraft installations. Faraday Effect causes light's polarization plane in a medium to rotate when the medium is exposed to a magnetic field in the direction of light propagation. Using optical fiber as the propagation medium and by forming closed fiber loops, measuring the total light rotation would result in the total current enclosed. It is noted that the sensing element is an optical fiber, thus termed "intrinsic" sensor. In contrast, in "extrinsic" sensor optical fiber is only used for relaying signal from a remote sensor.

This sensor overcomes many limitations of traditional sensors. Much like a Rogowski coil, total current is measured by installing fiber loops around the structure of interest. However, the sensor is self-integrating, thus no integrator needed, and DC component can be measured with suitable electronics. It does not suffer from self-resonance, hysteresis and saturation as with Rogowski coil or current transformers. It is not susceptible to arcing/sparking from high voltage and current. Measurement can be performed at arbitrary locations on the structure, unlike a resistive shunt that must electricallyisolate fuselage sections.

There are also many unique characteristics. It is small, light-weight, flexible, yet can safely and accurately measure very large lightning current. It is highly conformable to arbitrarily complex structure shapes, and can fit tightly onto structure's surfaces. Installation is simple and non-intrusive, simply by wrapping the thin fiber one or more times around the structure to be measured. Being non-conductive, the sensing fiber can be safely routed directly into the aircraft fuselage, building, or through flammable regions without requiring special converters. Versatility is excellent, as the same fiber can be used on both small and large structures, given sufficient length and bandwidth. Measurement sensitivity may be tailored by varying the number of fiber-turns around the conductor.

The sensor is not without limitations. Fiber choice is limited, as most commonly available fibers are based on silica in which the Faraday Effect is weak. However, the weak effect makes the sensor highly suitable for large current applications such as lightning measurement. There are slight sensitivities to bend/vibration and temperature variations, but there are compensation methods. Glass fiber can be fragile and needs suitable protection. 
Aircraft installations and their demanding safety and aerodynamic performance requirements would benefit the most from this new sensor's many unique characteristics previously described. Flight measurements would be greatly simplified by the direct measurement of total current; no longer required are field sensors and analytical efforts necessary to solve an inverse problem. Fig. 1 illustrates fiber-optic sensor loops installation in contrast with traditional B-Dot sensors that can only measure local magnetic fields.

To date the sensor has not yet been flown on an aircraft due to high costs in any flight experiment. In addition, most aircraft avoid flying into thunderstorms intentionally due to damage risks. Regardless, future flight efforts could significantly benefit from the sensor's simplicity, accuracy and the direct current measurement ability. The sensor's many advantages could also benefit other direct lightning measurements such as on small buildings, windmills, or lightning towers.

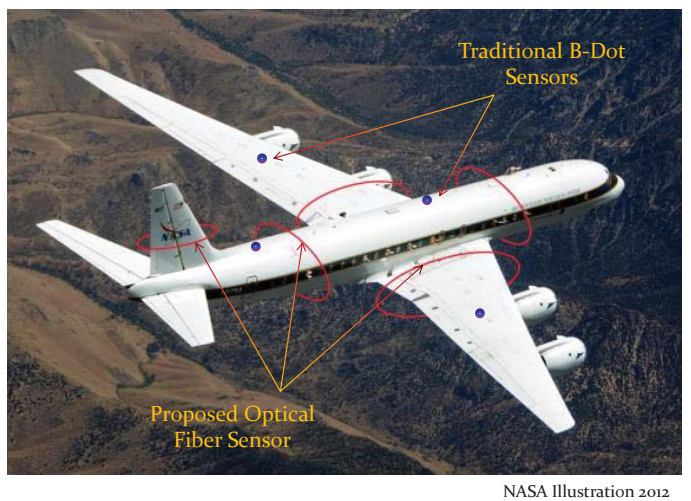

Figure 1. Optical-fiber sensors aircraft installation.

Current sensing using optical fiber has been under development for decades. However, commercial products are developed mostly for measuring electrical power and may not be suitable for higher bandwidths in lightning. The approach presented this paper is tailored for direct lightning measurement and provides reasonable compromises between bandwidth, measurement range and accuracy. In addition, the design and implementation approach gives emphasis to measurement stability, simplicity, low-cost, the ability to measure (near) DC component in continuing current, and for not requiring highly specialized optical tools.

The remainder of the paper describes the sensor concept and implementation, laboratory testing, and field testing with rocket-triggered lightning. Some aircraft installation considerations and testing are also discussed. Two similar systems discussed in this paper are based on 1310nm and 1550nm laser wavelengths. The measurement range achieved is approximately $300 \mathrm{~A}-300 \mathrm{kA}$ for the $1310 \mathrm{~nm}$ system or 400 A - $400 \mathrm{kA}$ for the $1550 \mathrm{~nm}$ system, a $60 \mathrm{~dB}$ range. Both systems are less sensitive but otherwise similar to a $850 \mathrm{~nm}$ system that successfully measured rocket-triggered lighting [3].

\section{FiBER-Optic CURRENT SENSOR CONCEPT}

\section{A. Basic Sensor Operation}

The Faraday Effect causes linearly polarized light in the sensing fiber to rotate when exposed to an external magnetic field in the direction of light propagation [4-8]. The effect in an optical fiber is illustrated in Fig. 2. The total polarization plane rotation $\phi$ is

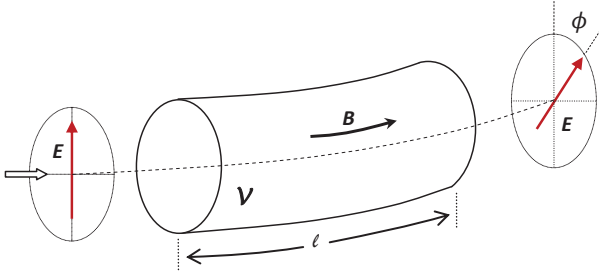

Figure 2. Faraday Effect in optical fiber.

$$
\phi=V \int \boldsymbol{B} \cdot d \boldsymbol{l}=\mu_{0} V \int \boldsymbol{H} \cdot d \boldsymbol{l},
$$

where $\mu_{0}$ is the free-space permeability; $V$ is the Verdet constant in radians/(meter-Tesla); $\mu_{0} V$ is the combined permeability Verdet constant (radians/ampere); $\boldsymbol{B}$ is magnetic flux density in Tesla (T); $\boldsymbol{H}$ is the magnetic field (amperes/meter); and $l$ (in meters) is the light and magnetic field interaction path length. Forming $N$ fiber loops and applying Ampere's law yields result in $\phi$ being directly proportional to the total current enclosed $I$ (Eq. 2). Thus, $I$ can be determined by measuring $\phi$. As shown in Eq. 2, the sensor is self-integrating, no additonal integration is needed.

$$
\begin{aligned}
\phi & =\mu_{0} V \oint \boldsymbol{H} \cdot d \boldsymbol{l}, \\
& =\mu_{0} V N I .
\end{aligned}
$$

\section{B. Polarimetric Detection Scheme}

The scheme to measure polarization change induced by current is illustrated in Fig. 3. Linearly polarized light is launched from a super-luminescence diode (SLD) broadband laser onto the sensing fiber at 4 that forms closed loops around a structure of interest. As light reflects from the Faraday Mirror at 6, its polarization is rotated 90 degrees relative to the incident light. This Faraday mirror implementation makes the system less sensitive to bending, since stress induced effects on polarization in one direction are canceled by the same effects on the orthogonal polarization in the reverse direction [5]. As reflected light traces back to the non-polarizing beam splitter (NBS) at 3, half of the power is reflected toward the half-wave plate (HWP) at 7 and the polarizing beam splitter (PBS) at 8 . Alternately, the PBS can be implemented in a fiber assembly. Exiting the PBS, light power in the two orthogonal polarizations are measured by two photo-detectors D1 and D2 at 9. The difference in optical powers at 9 is measured with a balanced detector, whose output voltage being proportional the difference between the two detectors' outputs. The HWP at 7 allows one to rotate and align the beam polarization incident on the PBS. 
Ideally, at zero current the incident polarization should be at $45^{\circ}$ relative to the PBS's two orthogonal principle polarization axes, so that beam power is divided equally between the two optical detectors at 9. With no current the balanced detector's output should be at zero volts. With current, Faraday Effect causes the light's polarization to rotate causing power imbalance at the two detectors, leading to a voltage output at the balanced detector.

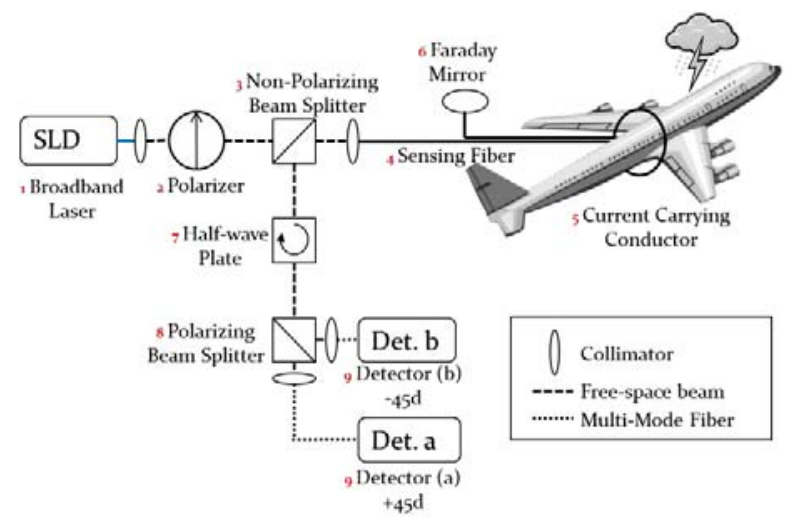

Figure 3. Opical detection scheme.

This setup is referred to as a reflective scheme since a mirror is incorporated and light travels round-trip. With the Faraday Effect being non-reciprocal, the Faraday rotation is doubled relative to single-pass scheme. The responses at the two detectors should ideally be (a),(b) $=0.5^{*}\left[1 \pm \sin \left(4 \mu_{0} V N I\right)\right]$ for a reflective scheme. Mathematic operation difference-oversum, $(c)=(a-b) /(a+b)$, yields

$$
\begin{aligned}
& (\mathrm{c})=\sin \left(4 \mu_{0} V N I\right), \text { or } \\
& N I=\sin ^{-1}(\mathrm{c}) /\left(4 \mu_{0} V\right),
\end{aligned}
$$

where equivalent current NI (in unit Ampere-turn) is defined as number of loops $N$ times the current $I$, and $\mu_{0} V=1.01 \mu \mathrm{rad} / \mathrm{A}$ at $1310 \mathrm{~nm}$ and $0.718 \mu \mathrm{rad} / \mathrm{A}$ at $1550 \mathrm{~nm}$ [4] for the two systems described in this paper.

Fig. 4 illustrates the ideal responses at 1310nm, with the response curves (a) and (b) being voltage outputs from the two optical detectors. The difference-over-sum operation, (c) $=(\mathrm{a}-$ $\mathrm{b}) /(\mathrm{a}+\mathrm{b})$, would yield a response that is more sensitive (higher slope) than either response curve (a) or (b), with zero crossing at zero current, and has larger dynamic range due to commonmode noise subtraction. Current $I$ or equivalent current $N I$ is then computed from (c) using eq. (4).

The typical operating range is where the curve (c) increases monotonically, or about $-350 \mathrm{kA}$ to $+350 \mathrm{kA}$. In this range the response and current correspond one-to-one. In the systems described, the non-ideal fiber medium and optical components distort the curves. The practical range is slightly reduced to about $-300 \mathrm{kA}$ to $+300 \mathrm{kA}$, as shown in the next section.

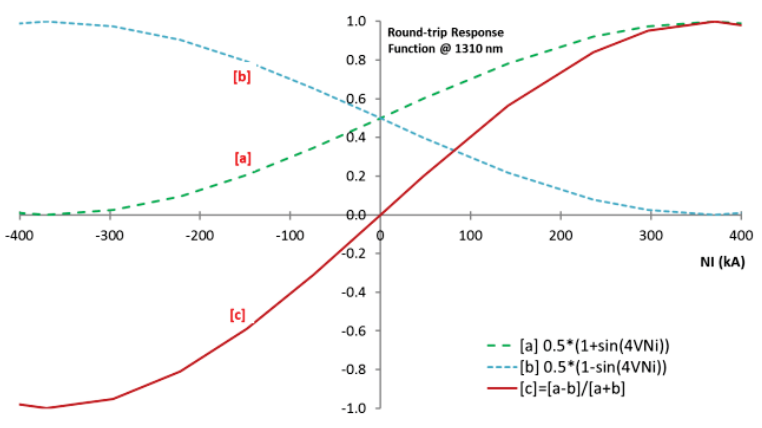

Figure 4. Ideal sensor responses at 1310nm.

The $1550 \mathrm{~nm}$-based system is slightly less sensitive due to the lower Verdet constant at this wavelength, so it can measure slightly larger current. The practical range is approximately +/$400 \mathrm{kA}$. The design, construction and characteristics otherwise are very similar to the $1310 \mathrm{~nm}$ system.

Over-current would not damage the sensor. Light polarization would simply rotate beyond the intended range, and the solution to the $\sin ^{-1}$ function in (4) would be ambiguous. However, there are minor variations of the detection scheme that permit measurement of very large current [6]. These practices are not necessary here since for most direct lightning are within the $300 \mathrm{kA}$ and $400 \mathrm{kA}$ range of the two systems described.

It is important that light's state-of-polarization (linear) is maintained in the fiber during light transit. This is achieved with fiber selection. The two systems in this paper use two different commercial highly spun polarization-maintaining (PM) fibers [7], which are the result of twisting PM fibers during manufacturing. Fiber twisting helps hold the state-ofpolarization that otherwise would be destroyed in a typical fiber. The twist rate is about $4-5 \mathrm{~mm}$ per turn. Additional information on fiber-optic current sensor can be found in [4-8].

\section{Sensor Calibration and Data Correction}

Calibration is performed in laboratory by relating the sensor's response curve to data from reference sensors, and a calibration function is developed. The reference sensors used include a Rogowski coil with an electronic integrator and a ferrite-based Pearson ${ }^{\mathrm{TM}}$ current transformer (CT). Fig. 5 compares the three sensors by plotting peak equivalent current using the Faraday sensor on the vertical axes against currents from the reference sensors on the horizontal axes. Large equivalent currents in the figure were achieved by using multiple fiber turns and/or a wire coil - details are described in the next section.

Ideally the Faraday sensor data would fall on the straight diagonal line labeled "ideal" representing (1:1) correspondence with the two reference sensors. Instead, the data follow the curve labeled "uncorrected". This non-ideal response is due to the reduced sensitivity in the spun fiber [7], relative to ideal medium of the same material - along with some light depolarization from the non-ideal fiber medium and optical 
components. Thus, it is important that the sensor is calibrated to develop a correction factor or function.

A correction function can be developed by spline-fitting the "uncorrected" curve in Fig. 5 with a polynomial function (typically $5^{\text {th }}$ or higher, odd order). The purpose is to map the Faraday sensor response curve to the "ideal" curve. That function is then applied to subsequent measurements to achieve the corrected results. Fig. 5 shows the "corrected" response curve aligns well with the "ideal" diagonal line. A $9^{\text {th }}$ order polynomial was used. Data interpolation may also be used instead of curve fitting; however, neither approach is perfect, and some small error may remain. In addition, only a simple correction factor may be needed if measurement is restricted to the linear region, i.e. $100 \mathrm{kA}$ or less.

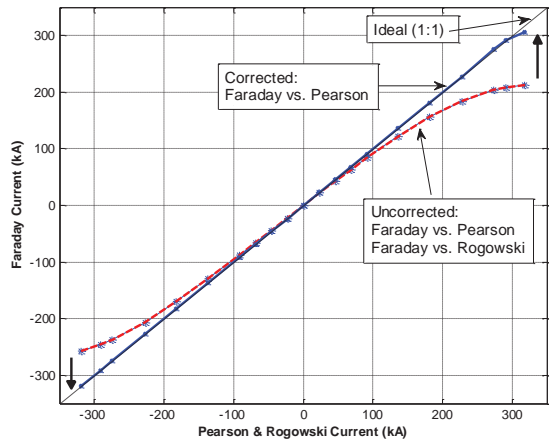

Figure 5. Example of corrected and un-corrected response curves.

\section{LABORATORY DEMONSTRATIONS}

This section describes various laboratory lightning measurement demonstrations. The measurements include a) simulated large current using multiple fiber loops and/or a current coil, b) up to $200 \mathrm{kA}$ direct lightning standard test waveform performed at a commercial laboratory, c) current on a simulated aircraft fuselage, and d) low-level indirect lighting current inside a simulated fuselage. Demonstrations measuring rocket-triggered-lightning are described in the next section.

\section{A. Laboratory Simulated Large Current}

It is difficult and unsafe to achieve in laboratory settings current levels up to $300 \mathrm{kA}$ (or $400 \mathrm{kA}$ for the $1550 \mathrm{~nm}$ system) for the purpose of calibrating and testing the sensor. One acceptable approach to producing the associated optical effects in the fiber is by using multiple fiber loops and/or a multi-turn conductor coil. Multiple fiber loops and/or multi-turn coil amplify the Faraday rotation beyond the amount produced by a single fiber loop around a single conductor. The amplification factor is the multiplication of the number of fiber-loops and the number of wire turns used. Fig. 6 illustrates a typical setup. Laboratory tests show using multiple fiber loops, a multi-turn coil, or combinations of both yield the same response curves. Using a wire coil having a high number of turns may distort the injected waveform due to the increased inductance. In contrast, a high number of fiber loops does not affect the current waveform but would require a longer sensing fiber.
For simplicity, $N$ is redefined from Eq. (4) to be the product of the number of fiber loops and the number of wire turns. The product $N I$ is referred to as equivalent current as previously stated in Eq. (4).

Fig. 7 illustrates results comparison between the Faraday sensor and the reference sensors, with different number of fiber turns and wire loops. The equivalent currents $N I$ are about 5 $\mathrm{kA} \bullet$ turns and $300 \mathrm{kA} \bullet$ turns. The same calibration correction function was used and good results were achieved in both cases. Similarly, NI up to $400 \mathrm{kA} \bullet$ turns was demonstrated with the $1550 \mathrm{~nm}$ wavelength system with equally good results. Since the reference Rogowski coil and Pearson current transformer (CT) only measure current on one wire-turn, their results are numerically scaled up by the factor $N$ for the comparison. This practice is commonly used and accepted [48].

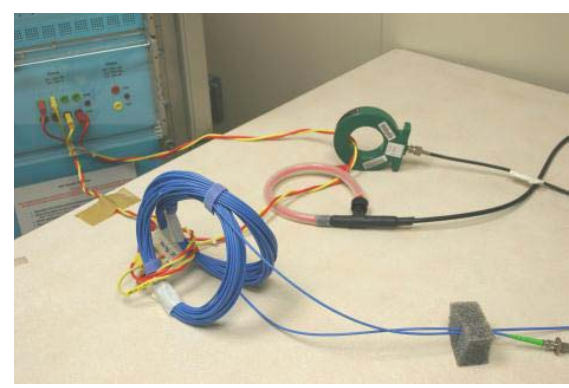

Figure 6. Using multi-turn coil and multiple fiber loops to achieve high current effects.
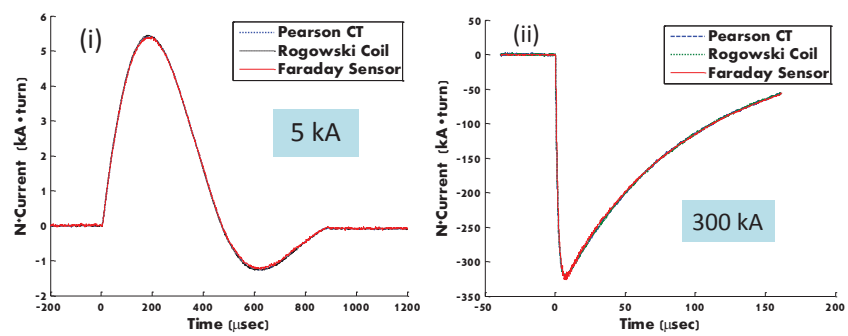

Figure 7. Laboratory testing at $N I=5 \mathrm{kA}$ and $300 \mathrm{kA}$. (i) 49-turn coil and one fiber loop ( $\mathrm{N}=49)$, and (ii) 3-turn coil and 28 fiber loops $(\mathrm{N}=3 * 28=84)$.

\section{B. Direct Lightning Large Current Measurement}

The 1310nm sensor system was evaluated for large current performance using only one fiber loop around one conductor $(N=1)$. Using one fiber loop would be similar to installation external to an aircraft fuselage, a large structure, or a lightning tower. The tests were performed at a commercial lightning test facility using standard aircraft lightning test waveforms and include components D, B and C [9]. Test current amplitudes were 20, 40, $100 \mathrm{kA}$ with double-exponential waveforms. In addition, $200 \mathrm{kA}$ tests with damped sinusoidal waveform were also conducted. This was necessary due to the test facility's limited abilities to generate unipolar waveforms at high current. The measurement piggybacked on a separate effort to evaluate lightning effects on composite panels. 
The setup shown in Fig. 8 included the fiber forming one closed loop around a flat metal plate (about $0.5 \mathrm{~m}$ wide) carrying the return currents that exit the composite panels under test. Due cable routing restrictions, a section of the fiber was routed near the lightning test zone on its way to the optical box. Closing the fiber loop at the optical box to achieve good measurement isolation was not possible; thus, some error was anticipated. The Faraday sensor results were compared against the sum of four Pearson CTs measuring currents exiting the four sides of the composite panel.

Fig. 9 shows results for $100 \mathrm{kA}$ and $200 \mathrm{kA}$ peak current. The comparisons are reasonably good considering the nonoptimal setup. The errors are about 3-10\% depending on the routing of the exposed fiber section through the lightning test zone. It is clear that the Faraday sensor is capable of directly measuring $200 \mathrm{kA}$ current using just one loop, and that a better setup could lead to better comparisons. The $20 \mathrm{kA}$ and $40 \mathrm{kA}$ comparisons are similar to the $100 \mathrm{kA}$ results.
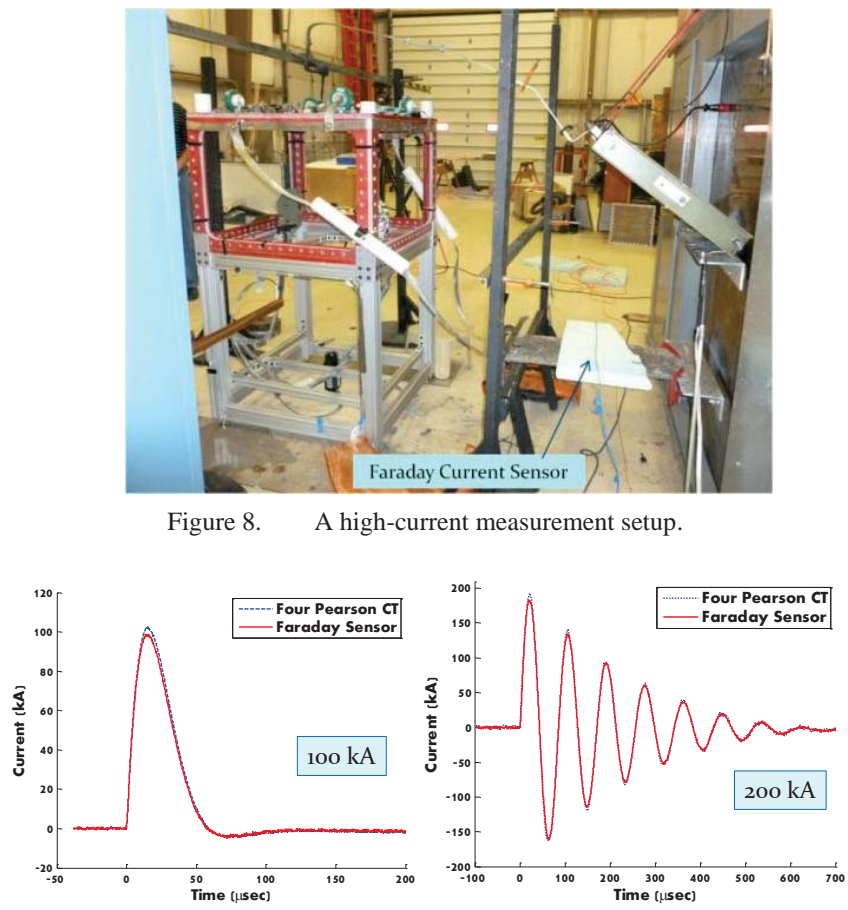

Figure 9. Measurements of $100 \mathrm{kA}$ and $200 \mathrm{kA}$ on a current return plate.

\section{Measurement on Simulated Fuselage}

Fig. 10 illustrates a setup measuring current on a $1.2 \mathrm{~m}$ diameter aluminum cylinder that simulates an aircraft fuselage or round lightning tower. Current lightning waveforms from 250 A to $4 \mathrm{kA}$ (amplitudes limited by laboratory equipment) were injected onto the cylinder at the bottom left location. Return currents were extracted from the cylinder at bottom right.

The $15 \mathrm{~m}$ sensing fiber form a single loop around the cylinder, with both ends co-routed to the optical box located $4 \mathrm{~m}$ away in the foreground. As shown in Fig. 10, the fiber closed the loop at the optical box without any unpaired fiber section, thus good isolation was achieved. A Pearson CT and a Rogowski coil provide reference comparison data. Fig. 11 shows good results for both the $250 \mathrm{~A}$ and $4 \mathrm{kA}$ tests.

Noise is observable in the 250 A measurement, illustrating the low level sensitivity limit. The dominant source of noise is the SLD laser, which is inherent a wideband optical noise source. Noise reduction techniques implemented include using the balanced detector for common mode noise subtraction and a $1.9 \mathrm{MHz}$ low-pass filter. In addition, a small movingwindow data averaging is implemented in post-processing, i.e. 11-point window out of 10,000-points data length. A $60 \mathrm{~dB}$ range could be achieved with this setup.

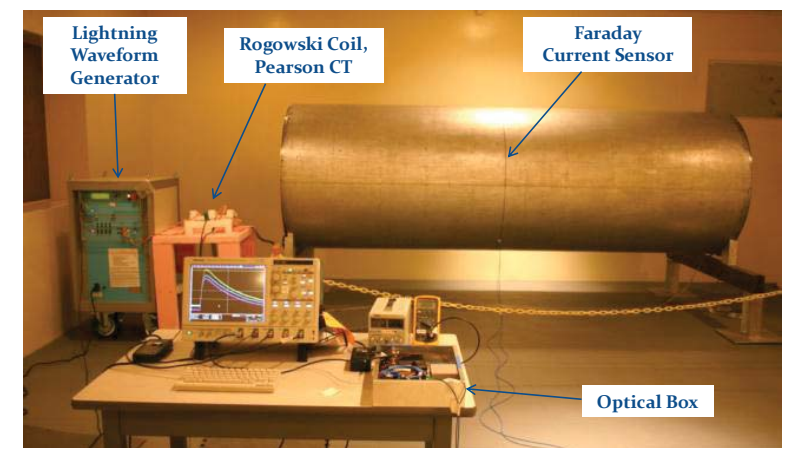

Figure 10. Measurement on a 1.2m diameter aluminum cylinder simulating an aircraft fuselage.
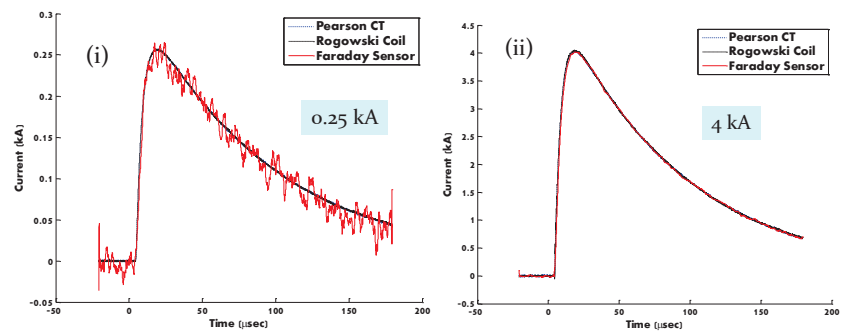

Figure 11. 250A and 4 kA peak current measurements on a $1.2 \mathrm{~m}$ (4 feet) diameter structure.

\section{Indirect Lightning Internal Current Measurement}

Figs. 12-13 demonstrate excellent isolation for low level current measurement on an internal structure. This setup simulates induced current on equipment or wire bundles in the presence of significantly larger current flowing on the outer aluminum structure. About $2 \mathrm{kA}$ peak total current was injected on the aluminum cylinder, with about 140 A flows on the internal box. With some slight data averaging for noise reduction, generally good comparison can be observed even though the Faraday sensor's data were close to the noise floor.

Excellent results were also performed when the fiber loop was expanded all the way to the metal frame of the aluminum structure. This illustrates the ability to measure the composite sum of all lightning induced current on all internal cables and structures. This ability could precisely determine the occurrence of strong lightning induced current, and help with equipment maintenance scheduling after a lightning strike. 


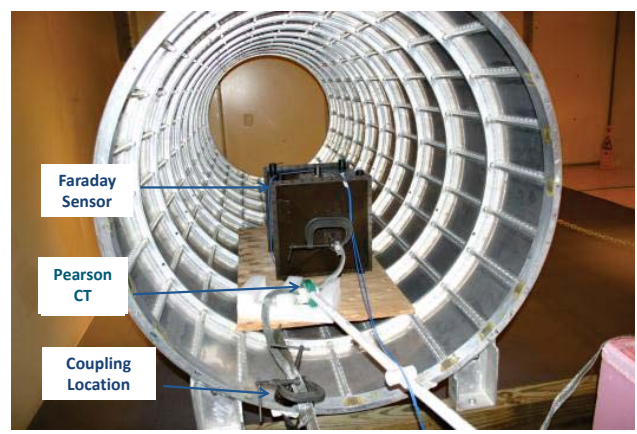

Figure 12. Low-level measurement on an internal structure.

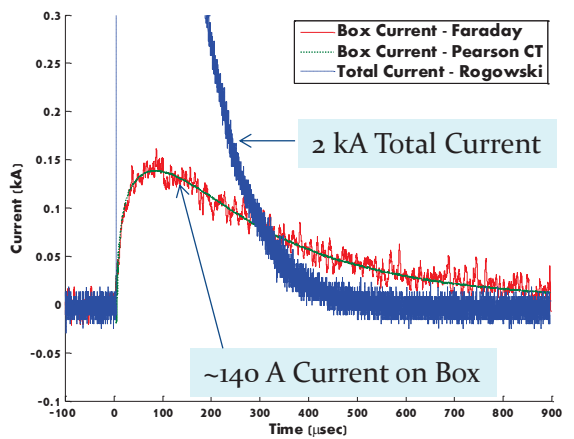

Figure 13. Good comparison for low-level measurement in presence of larger current on an external structure.

\section{Rocket-Trigged Lightning MEAsurement}

The $1550 \mathrm{~nm}$ system ( $N I=400 \mathrm{~A}-400 \mathrm{kA}$ range) was demonstrated measuring rocket-triggered lightning in 2012. The measurements were performed at the International Center for Lightning Research and Testing (ICLRT) at Camp Blanding, Florida, U.S.A. facility [10]. The measurements were similar to an earlier (2011) successful effort that used twisted single-mode sensing fiber [3] at 850nm wavelength.

In the setup shown in Fig. 14, triggered-lightning flashes would attach to the intercepting ring, and lightning current would travel to the ground via a down-conductor. A resistive shunt (T\&M Model R-7000-10) in-line with the down conductor provided reference measurements. The sensing fiber formed four loops around the same down-conductor. The remaining fiber segments at the two ends were co-routed radially away from the site. One end was connected to the supporting optical box $12 \mathrm{~m}$ away. The other end was connected to a Faraday mirror $4 \mathrm{~m}$ away that was buried in the ground to minimize temperature variations. Data were recorded using a commercial 14-bit digitizer sampling at 100 MHz. The sensors and digitizers were powered by batteries.

Due to insufficient fiber length, the Faraday mirror was not positioned near the optical box. About 8m (from the Faraday mirror to the optical box) section of the sensing fiber was "unpaired", potentially exposed to effects from the lightning flash and strong ground currents that would normally be canceled with a closed loop. However, by routing the fiber in the radial direction away from the site, magnetic field components in the direction of the fiber are expected to be minimized, reducing any undesirable effects. The fiber was also protected from wild animals or being trampled on inside combinations of rain gutters and plastic braided sleeves.

The system successfully measured rocket-triggered lightning with excellent result comparisons. With the four fiber loops $(N=4)$ used, the measurement range for the sensor is $100 \mathrm{~A}-100 \mathrm{kA}$. Fig. 15 illustrates the representative results. Also shown is low-level comparison to highlight the measurement range and the ability to measure continuing current. Data were slightly smoothed for noise reduction. The results are nearly identical between the two sensors. Minor peak amplitude differences in some data (up to 500A) were attributable to interference coupling from ground lightning current. The good results illustrate the system can successfully operate in realistic lightning environments. Additional sensor validation data can be found in [11-12].
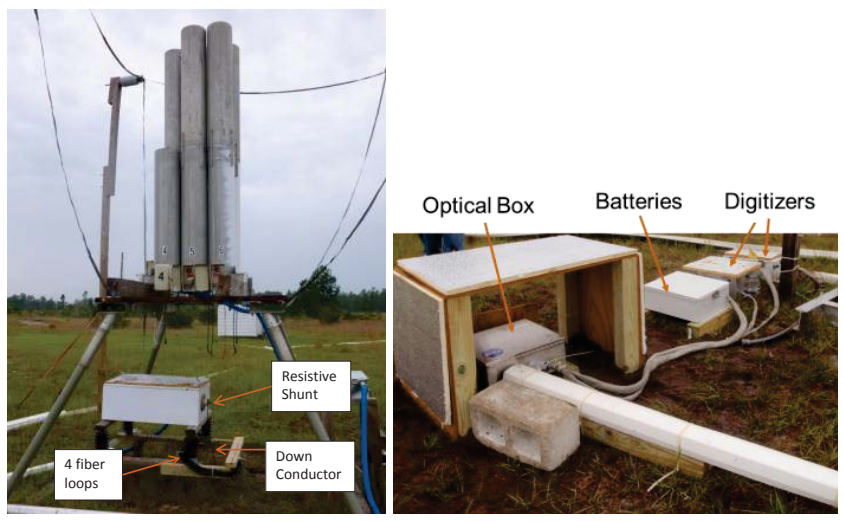

Figure 14. Rocket-triggered lightning current measurement setup at ICLRT.
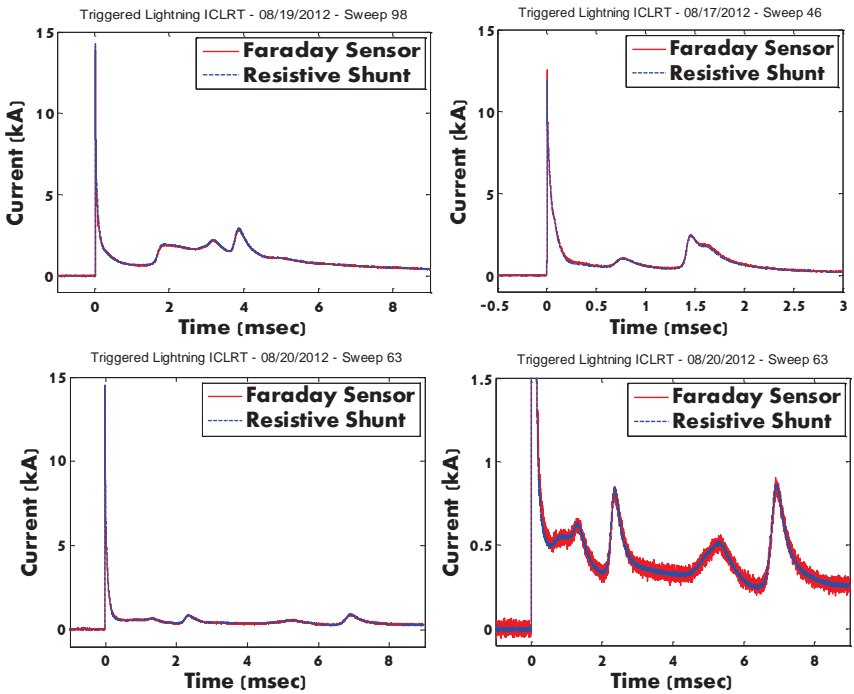

Figure 15. Sample triggered lightning measurements results and low-level comparison. 


\section{AIRCRAFT INSTALLATION AND CONSIDERATIONS}

It is a goal to fly one or more sensors to collect aircraft lightning data. A basic multi-sensors setup is illustrated in Fig. 16 with four sensing fiber loops along with optical interface boxes. Sensor outputs are recorded using a high speed data recorder. In addition, AC components are recorded separately and used for recorder triggering (more details later). No additional optical/electrical interface is needed as the sensing fiber is inherently safe and can be routed directly into the aircraft. No other field sensors are necessary to trigger the system. Installation is relatively simple due to optical fiber's small size and weight. However, there are a few considerations to be discussed below.

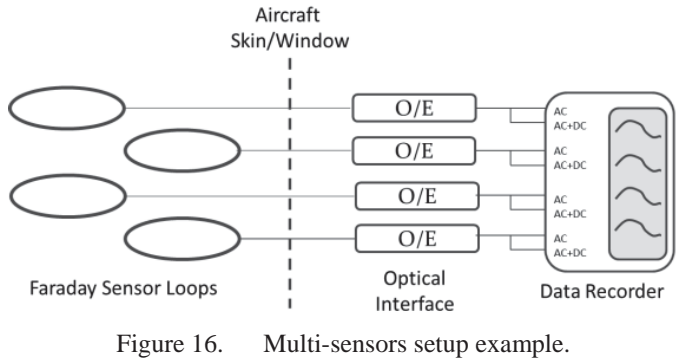

\section{A. The Sensor and Data Acquisition Systems}

Of three systems constructed and tested operating at $850 \mathrm{~nm}$ [3], $1310 \mathrm{~nm}$ and $1550 \mathrm{~nm}$, the $1310 \mathrm{~nm}$ system is chosen for future aircraft installations. This wavelength provides slightly better sensitivity than the 1550nm (300 A vs. $400 \mathrm{~A}$ ) while offers adequate measurement ceiling at $300 \mathrm{kA}$. In addition, optical components are readily available and can be smaller than for the $850 \mathrm{~nm}$ system. Other wavelengths are also possible though not tested in this effort.

The 1310 system was re-configured for aircraft installation as shown Fig. 17. Digital displays were added for health monitoring. The system is fitted with a $40 \mathrm{~m}$ long spun fiber for installation flexibility. Though multiple sensors are envisioned in future setups, only one system is planned for the initial test.

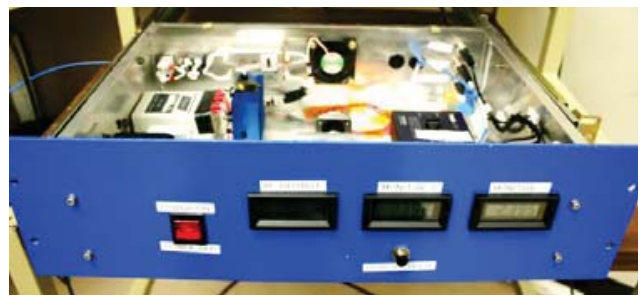

Figure 17. Faraday-sensor flight optical box.

The sensor's output is recorded using a commercial digitizer/recorder capable of 100 mega-samples per second at 14-bit data length. Up to 20 data channels (using 5 data acquisition boards) can be recorded into the system's solid state memory.
Data recording is triggered on the AC component of the output. This is more accurate than triggering on the total current due to a slow and small DC voltage drift caused by various environmental factors. In the $1310 \mathrm{~nm}$ system the voltage drift can be up to an equivalent of $600 \mathrm{~A}$. The DC drift can be subtracted out at data post processing by comparing against baseline data just before the lightning strike.

\section{B. Fiber Installation Locations}

The primary fiber loop locations are around the fuselage before and after the aircraft's wing section. These two locations would capture most of lightning current paths except for the wing-to-wing path. And by having the fiber loop close to the wing section more of swept-stroke lightning components may be captured. Locations on aircraft wings are also desirable; however, moving surfaces and landing gears could be problematic on some aircraft. A small metal panel in place of a window could provide multiple fiber penetrations into the fuselage.

\section{Engine Ingestion and Adhesive Tape Testing}

Engine ingestion is a significant concern if the fiber is installed in front of engine intakes. This is the case for aircraft with engines mounted toward the rear section. The fiber, therefore, must be properly secured, possibly with adhesive tape and non-metallic clamps.

To develop confidence in the installation method and the adhesive tape, representative fiber sections were flown on two different aircraft. Aircraft-grade polyurethane tapes were used. One installation, Fig. 18, has been flown for over a year and includes short metal wire sections (in place of fiber) in three orientations. The other installation includes a representative fiber loop fully encircling the aircraft fuselage at location behind the engine intakes. This second setup shown in Fig. 19 has been flown for about six months. Neither setup has shown signs of degradation to date. Successful tests would minimize concerns for engine ingestion and would allow for the fiber installation at locations in front of engine intakes.

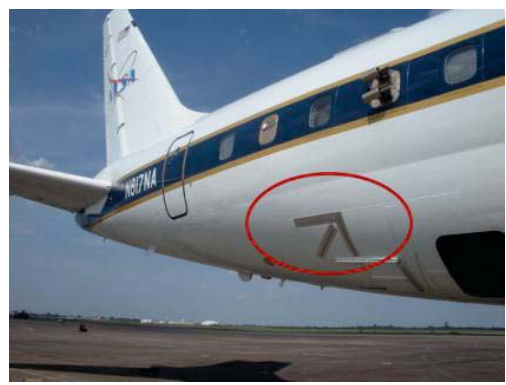

Figure 18. Adhesive tape testing holding wire samples in three polarizations (circled). 

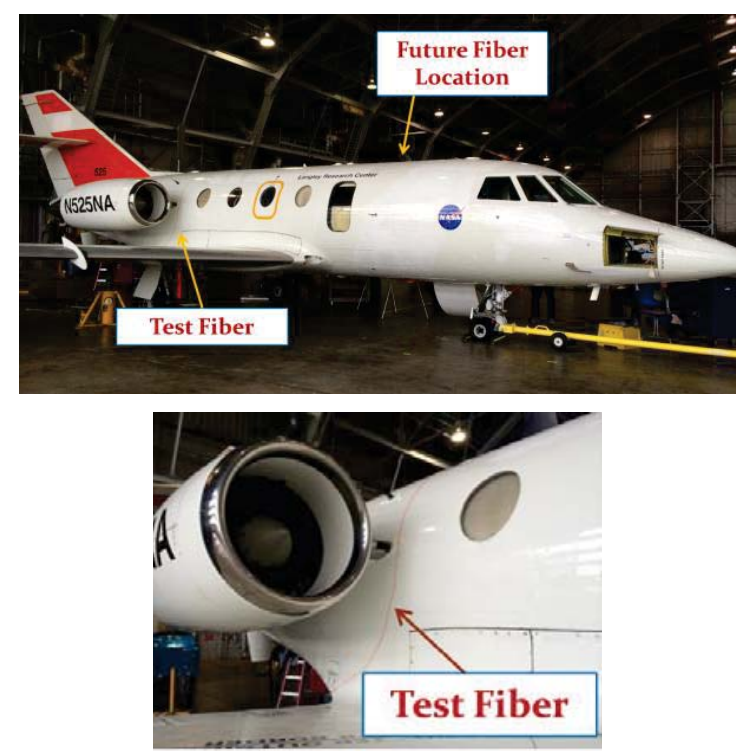

Figure 19. Adhesive tape holding test fiber loop around the fuselage.

\section{Equipment Shielding}

The optical interface box, data recorder and cabling could potentially be affected by strong magnetic field produced by lightning current if installed near windows. This need is evidenced in Section IV where the supporting equipment and cabling were affected by lightning ground current. A shielding enclosure or magnetic shielding materials installed at nearby windows could minimize the concern.

\section{CONCLUSION}

The design, accuracy, advantages and versatility of fiberoptic current sensor were validated though multiple demonstrations. The demonstrations included measurements of large current up to $200 \mathrm{kA}$, current on and inside a simulated fuselage, and triggered-lightning current. The sensor's many advantages make it unique for many lightning measurement applications.

\section{REFERENCES}

[1] P. Laroche, P. Blanchet, A. Delannoy, F. Issac, "Experimental Studies of Lightning Strikes to Aircraft," Onera Aerospace Lab Journal, Issue 5, December 2012 (AL05-06).

[2] F. Pitts, B. Fisher, V. Mazur, and R. Perala, "Aircraft Jolts from Lightning Bolts," IEEE Spectrum, July 1988.

[3] T. Nguyen, J. Ely, G. Szatkowski, C. Mata, A. Mata, G. Snyder, "FiberOptic Sensor for Aircraft Lightning Current Measurement," 2012 Int. Conf. on Lightning Protection (ICLP).

[4] J. M. Lopex-Higuera, Editor. Handbook of Optical Fibre Sensing Technology, 2002; Sections 27.2 - 27.4.

[5] P. Drexler and P. Fiala,"Utilization of Faraday Mirror in Fiber Optic Current Sensors", Radioengineering, Vol. 17, Dec. 2008.

[6] A. White, G. McHale, D. Goerz, "Advances in Optical Fiber-Based Faraday Rotation Diagnostics," 17th IEEE Int. Pulsed Power Conference, Wash. DC, July 2009 (LLNL-CONF-415198).

[7] R. Laming and D. Payne, "Electric Current Sensors Employing Spun Highly Birefringent Optical Fibers," Journal of Lightwave Technology, Dec. 1989.

[8] G.W. Day, M.N. Deeter and A.H. Rose, "Faraday effect sensors: A review of recent progress," Proc. SPIE 1779, 1992, pp. 21-26.

[9] ARP-5412 "Aircraft Lightning Environment and Related Test Waveforms," Rev. B, Jan 2012.

[10] V. Rakov, "A review of Triggered-Lightning Experiments," 30th International Conference on Lightning Protection, Cagliari, Italy, September 13-17, 2010. Update!

[11] T. Nguyen, J. Ely, G. Szatkowski, C. Mata, A. Mata, and G. Snyder, "Fiber-Optic Current Sensor Validation with Triggered Lightning Measurements," 2013 Int. Conf. on Lightning and Static Electricity (ICOLSE)

[12] T. Nguyen, J. Ely, G. Szatkowski, C. Mata, A. Mata, G. Snyder, “An Intrinsic Fiber-Optic Sensor for Structure Lightning Current Measurement," 2014 Int. Lightning Detection Conference (ILDC). 